de 2010, de http://www.undp.org/spanish/proddal/informeproddal.html

PNUD, PROGRAMA DE LAS NACIONES UNIDAS PARA EL DESARROLLO. (2008). Estado, democracia y construcción de ciudadanía en América Latina. Sintesis de los debates del Seminario Internacional "Democracia, Estado y Ciudadanía en América Latina". Recuperado el 4 de abril de 2010, de http://www.pnud cl/publicaciones/Debates\%20Seminario\%20Democra-
cia\%20AL.PDF.

RACZYNSKI, D. y SERRANO, C. (2005). Las políticas y estrategias de desarrollo social. Aportes de los años 90 y desafios futuros. En: Meller, P. (ed.) La paradoja Santiago de Chile: Taurus.

RAMOS, M. y GUZMÁN, J. (2000). La guerra y la paz ciudadana. (la. ed.) Santiago: LOM.

ROBLEDO, A. y RODRÍGUEZ, P. (2008). Emergencia del sujeto excluido: aproximación genealógica a la no-ciudad en Bogotá. (1a. ed.). Bogotá: Ediciones Pontifici Universidad Javeriana.

SALVAT, P. (2002). El porvenir de la equidad. Aportaciones para un giro etico en la filosofía política contemporánea. (la. ed.). Santiago: LOM.

VELÁSQUEZ, E. (2002). Historia de la doctrina de la seguridad nacional. Revista Convergencia, 9, 27, 11-39.

WACQUANT, L. (2001). Parias urbanos. Marginalidad en la ciudad a comienzos del milenio. (2a. ed.). Buenos Aires: Manantial.

WACQUANT, L. (2008). Urban outcasts: a comparative sociology of advanced marginality. (la. ed.). Cambridge: Polity Press.

\title{
Organizaciones del Tercer Sector: avanzando en la necesidad de caracterizar al voluntariado chileno
}

Organizations of the Third Sector: advancing in the need to characterize the Chilean voluntary work

RODRIGO FLORES G.

Fscuela de Trabajo Social,Pontificia Universidad Católica de Chile.Correo electrónico: rfloresu@uc.cl

María Ignacia Donoso $S$.

Programa de Magister

Correo electrónico: mdonoso6@uc.d

\section{Resumen}

El voluntariado se constituye hoy, como una de las más importantes iniciativas de participación ciudadana. De igual, manera es un elemento clave dentro de las organizaciones sociales en la medida en que aportan a la creación de ventajas competitivas, transformándose en activos de gran valor. Su creciente complejización y diferenciación, plantea nuevos retos a las organizaciones sociales en términos de mejoras organizativas y gestión de calidad, identificándose la necesidad de contar con perfiles de voluntarios como un factor clave para lograrlo. Es en este contexto, donde

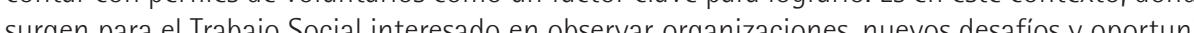
dades, aportando en dades, apotando en la formu able abordaje.

Palabras clave. Sociedad Compleja, Organizaciones Sociales, Perfil del Voluntariado.

Abstract

The voluntary work is constituted today, as one of the most important initiatives of civil participation. It is also a key element in the social organizations, because they contribute to the creation of competitive advantages becoming actives of great value The increasing complexity and differentiation, raises new challenges to the sociat organizations in terms of organizationa improferents and auses new managengt, for that it is necessary to count with a volunteering characterizationd a key It is in this context where there aise new challent with a volunteen for Social Work

Key words. Complex Society, Social Organizations, Volunteering profile.

Introducción

Organizaciones del tercer sector

Las organizaciones se constituyen en sistemas caracteristicos de la sociedad compleja, relacionándose con ella de tal manera que algunas se forman a partir de los sistemas de funciones de la sociedad, asumiendo su correspondiente primado funcional, buscando soluciones específicas a los problemas que deben afrontar. De esta manera, la economía es asumida como temática comunicacional propia de as empresas, la educación en los establecimientos educacionales, la salud en los hospitales, la política en distintos partidos, la justicia en los juzgados, el arte en museos y galerías y las religiones en las iglesias y sus particularidades, manteniéndose así la condición de que cada uno se vuelve a diferenciar en otros tantos sistemas organizacionales y así sucesivamente. 
No obstante lo anterior, ninguna organización puede atraer hacia sí, y responder en forma satisfactoria al complejo primado funcional del sistema y ejecutarlo como si se tratase de operaciones propias. Este es el caso de las asociaciones de empresarios, quienes asumen el tol de representantes de la eco-

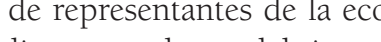
nomia, los colegios medicos para el caso del sistema salud o de las asociaciones de artistas, para el caso del arte. De este modo, los procesos políticos no se agotan en el sistema electoral democrático ni en la conformación de los partidos que intentan acceder al gobierno. Las esferas del poder trascienden el Estado, pero también en las decisiones de las organizaciones educativas se requiere imponer norma normas y reglamentos declarados sobre el proceso de enseñanza y isobre todo! de su aprobación. Ello deb ser objeto de observación y análisis, puesto que los sistemas de funciones surgen en base a la imposibilidad de organizarse a sí mismos (Luhmann, 1998). Dado que los problemas de la sociedad son complejos, difusos y no admiten especificaciones, las organizaciones públicas y privadas dejan parte importante de dichos problemas sin considerar. Esta es la razón por la cual el denominado tercer secto busca enfrentar problemas insuficientemente tratados por los otros sistemas organizacionales. Ante este panorama, el llamado Tercer Sector se posiciona como sistema capaz de entregar soluciones y de complementar las medidas del Estado y el Mercado. Hoy observanos que ONG y OSFL do. Hoy observamos que ONG y OSFL juegan un rol, inundando a la sociedad con comunicaciones de agenda pública, proponiendo metodologías no consideradas y actuando como articuladores de la ciudadanía (Abogair, 2006:9)

Las organizaciones sociales que forman parte del tercer sector son una de las más importantes formas de vinculación humana que existen en las sociedades contemporáneas. En su breve historia se han generalizado en todos los ámbitos, haciéndose cargo de operaciones sociales significativas por medio de clubes de ancianos, asociaciones de vecinos, sindicatos, centros de padres, fundaciones, corporaciones, cooperativas, etc Como elemento caracteristico, es posible observar en ellas elento caracteristico particularidades y diferencias con sistemas organizacionales que pretenden la busqueda del lucro aquellos que se encuentran extremadamente for malizados y pautados.

Otro tanto ocurre al analizar a sistemas organizacionales que se encuentran ligados a las demandas sociales sentidas por la población, fin social reparador o corrector. Este es el caso de las organizaciones de voluntariado. Desde nuestro punto de vista, ellas pueden operar con un código básico: inclusión / exclusión. Su particular forma de ejecución les permite convertirse en un verdadero puente ar culadicas y privadas de la sociedad. Este tipo de organizaciones sociales intentan encausar una serie de recursos -el tiempo, el conocimiento experto, infraestructuras de diversa índole, el dinero, etc.- hasta convertirlos en resultados que sean considerados beneficiosos para terceros, es decir, disminuir o mitigar una exclusión originaria (Flores, 2003).

Para el caso específico de este tipo de organizaciones, la membresía, en cuanto elemento funcionarial, es reemplazada por integrantes que se congregan de forma libre y voluntaria, inspirados en principios éticos y morales, generalmente de solidaridad y orientados preferentemente hacia la reciprocidad. Del conjunto de sus interacciones se genera una lógica de operación que se mueve en dos ejes: uno de ejes: uno de ellos orientado a la asistencialidad, la caridad y la reparación, generalmente económica, de la exclusión social. El otro, orientado a la reivindicación, la promoción o modificación y transformación social, por lo cual su ámbito de acción se concentra en el sistema político. Organizaciones no gubernamentales como la Cruz Roja Internacional, Médicos Sin Fronteras o las fundaciones son ejemplo del primer caso; mientras que sindicatos y ciertas organizaciones ecologistas son ejemplos del segundo.

De acuerdo a ciertas estimaciones, el sector no lucrativo chileno emplea en forma remunerada voluntaria sobre las 303 mil personas en Jornada Completa Equivalente (JCE). Si solo se considera el empleo remunerado, este representa al 2,6\% de la población económicamente activa. En otras palabras, la sociedad civil chilena emplea a más de tres veces el personal del sector minero $(1,3 \%)$ o a dos tercios del empleo de la construcción (8,1\%). También se puede expresar su importancia en el tamaño relativo del sector en términos de gastos, donde las instituciones sin fines de lucro representan un 1,5\% del PIB nacional (Salamon, 2006:5)

Al expresar el tamaño del sector no lucrativo en Chile, considerando el empleo total con respecto a la población económicamente activa, se obtiene que es más que el doble del tamaño observado en Brasil y Colombia. Si el tamaño del sector sin fines de lucro se mide solo atendiendo el empleo remunerado, Chile aparece inmediatamente des- pués de Argentina en cuanto a tamaño relativo en el contexto Latinoamericano, pero con una distancia considerable respecto de otros países del continente contemplados en el estudio, como son Perú, Colombia, Brasil y México (Salamon, 2006: 5).

Desde nuestra perspectiva, no cabe duda de que, a nivel societal, existe una especie de sobreproducción de expectativas respecto al quehacer y posibilidades de este tipo de organizaciones. Quienes con ellas tratan, trabajan o colaboran dejan de relacionarse de forma utilitaria para exigirles enunciados e imperativo ético-normativos. No es de extrañar, así, que se les exija convertirse en se les exija convirse en referentes o alten modelos y progams economicos y sociales actualmente vigentes. Ello, por cierto, no puede sino crear frustración y desesperanza en el corto plazo. Estas organizaciones, y la mayor parte de sus integrantes, no se encuentran orientados hacia tales fines. Es de sentido común que el cambio o la justicia social no pasa exclusivamente por el voluntariado.

\section{Las organizaciones de voluntariado}

No se desconoce el hecho de que algunas descripciones sociales sobre el voluntariado se encuentren teñidas de una idea esencialista y ontológica, donde su principal preocupación ha sido dar cuenta de lo que el voluntariado en realidad es y a quién es posible definir como voluntario (PNUD, 2003). Muy lejos de nuestra intención se encuentra el zanjar esta discusión, sin embargo, adoptamos la posición asumida por algunos autores (Davis, 1999) quienes requisitos, entre los que se pueden nombrar el no tener una recompensa material por la prestación de sus servicios, la libertad de involucramiento de su accionar, la consecución de beneficios que no son propios, sino altruistas y el carácter social de los emprendimientos propuestos. Tales características excluyen a la reciprocidad familiar.

En este sentido, es un lugar común sostener el declinar de lazos colaborativos, impulsados por una individualización creciente, donde el actor social, desprendido de comunicaciones aglutinantes como grupos de clase o religiosos, se ven impelidos a desarrollar acciones cuyas consecuencias no pueden ser sino sí mismos. Tales descripciones se explican como resultado del debilitamiento de instituciones tradicionales, fomentando el desinterés por asumir responsabilidades colectivas bajo ciertos supuestos ético-morales. Las actividades y vínculos ligados a desempeños altruistas, tales como la solidaridad, el voluntariado y la asociatividad, pueden definirse como excedentes sociales, altamente improbables o reaccionarios a las comunicaciones modernizadoras. Dichas descripciones van acompañadas de , de fomento de la sociedad civil y empoderamiento ciudadano. Su desmentido o refutación, aparece para el análisi social como un argumento tan básico y sencillo como la proposición que pretende contrariar, no aportando nada nuevo al conocimiento y comprensión del fenómeno.

Contradiciendo a los agoreros de principios de siglo, es posible observar un sostenido aumento en a cantidad de personas que desarrollan actividades de involucramiento voluntario en entidades privadas, corporaciones, fundaciones y agrupaciones de diverso tipo (Zulueta, 2003), las cuales incluyen actividades tan variopintas como recreativas y deportivas, organizaciones ciudadanas y funcionales, grupos de autoayuda y de ayuda filantrópica a terceros y en menor medida, movimientos sociales de carácter político. En este sentido, y de acuerdo a los datos arrojados por el Primer Catastro Nacional de Voluntariado (DOS, 2009), en Chile habría 1.277 entidades de voluntariado. Sin embargo, si se consideran las presencias comunales que estas mismas entidades tienen, se llega a la conclusión de les" (filiales, grupos, representaciones, etc.) ${ }^{3}$. Así, es posible afirmar la presencia de entidades de voluntariado en todas las comunas del país y de más de 204.594 voluntarios movilizados por ellas a nive nacional (DOS, 2009: 07).

En esta línea, estudios llevados a cabo indican que cuatro de cada diez encuestados (42\%) declara haber participado en alguna oportunidad en la realización de una tarea voluntaria. El mismo estudio sostiene que de cada cien personas, diecinueve han desarrollado algún tipo de actividad voluntaria en el último año (DOS, 2004a). Actualmente, el 91\% de las organizaciones chilenas del denominado Tercer sector tiene voluntarios (Olate, 2009).

La mayoría de las organizaciones de caridad no podría sobrevivir sin sus voluntarios, y ciertamente sería menos productivo responder sin ellos (Hager $\&$ Brudney, 2004: 12), aunque se constata la falencia de que, a parte de los programas de fomento del voluntariado de la DOS, no existen instancias y ofertas del Estado para la promoción del voluntariado. El trabajo voluntario se transforma, así, 
en característica propia de las organizaciones pertenecientes al Tercer Sector, en tanto es el rasgo principal que las diferencia de otro tipo de organizaciones, pero no es así únicamente porque las personas voluntarias constituyen un recurso importante, sino porque representan ideales y principios en en los cules se fuch voluntarios son capaces de dinamizar actividades innovadoras y descubrir necesidades no atendida en la sociedad que, con el tiempo, se convierten en actividades generadoras de empleo y riqueza para el conjunto de la sociedad, llegando a crearse sectores de actividad económica para las empresas, como es claro en el ámbito de los servicios sociales (Fundación Luis Vives, 2000:08).

Una de las características de importancia social que reviste el voluntariado es que se constituye como un ejercicio de ciudadanía y de participación, que toma su significado de los conceptos de solidaridad -en cuanto a los mecanismos distributivos y recíprocosy justicia -porque opera como un mecanismo de reparación de las diferencias. Se entiende que con l voluntariado se ejerce ciudadanía, en tanto esta implica la identificación del individuo como poseedor de derechos, entre los que destaca de modo principal el derecho a asumir libremente sus compromisos, manifestado en la voluntad de cooperar (Caritas, 2011: 9). De este modo, el voluntariado alienta la noción de una "ciudadanía ampliada" que cia los derechos económicos, sociales y culturales y funciona como un impulso para la acción democr tica y el desarrollo (Perold y Tapia; s/a: V-VI)

\section{Implicancias de la calidad para}

\section{las prestaciones que realizan las}

\section{organizaciones del tercer sector}

A nivel internacional, como también en nuestro país, muchas de las OSFL se han comprometido como socias estratégicas en las políticas sociales desplegadas por el Estado y han pasado de ser exclusivamente privadas a asociadas u operadores de programas del sistema público, debido a que se les reconoce su mayor capacidad para proveer servicios sociales, quedando en sus manos la gestión o administración directa de un porcentaje no menor de programas sociales (Vaughan \& Arsneault, 2003). Surge así la exigencia por la profesionalización, mejoras en su accionar, modernizar la gestión, esablecer métodos de transparencia y busq queda de estrategias que mejoren su sostenibilidad, crecimiento y eficiencia en los distintos campos en los cuales se desarrollan las intervenciones sociales con organizaciones (Coulshed, 1998).

Dada la creciente formalización que ha logrado el área de cooperación entre Estado y organizaciones privadas para el diseño y desarrollo de programas sociales, aumentan también las exigencias de calidad y transparencia en las acciones que desarrollan. Cabe también incluir los servicios y prestaciones que se les ofrece a los grupos más vulnerables de la población, de modo que ya no es suficiente que las organizaciones del tercer sector sean capaces de construir confianza, ahora también deben construir calidad (Vernis, 2005).

El concepto de calidad aplicada a distintas formas de intervenciones sociales implica considerar el paso de dicha noción desde la industria a los servicios sociales. Su uso original proviene del ámbito de la gestión y su énfasis se encuentra en el cumplimiento continuo de estándares consensuados. Algunas veces el término se ha referido a los productos de la organización, en otras a los procesos mediante los cules se obtenín esos productos, oras a la vida de las personas que trabajan en ella y, por último, al funcionamiento y orientación global de toda la organización. En todos estos significados a veces se ha adoptado un carácter más estático y otras más dinámico (Quijano, Cornejo, Yepes \& Flores, 2005).

La búsqueda de calidad en las intervenciones sociales que realizan las OSFL que trabajan con personas, puede definirse como una práctica, un modo de hacer que aporta valor añadido a los resultados de la organización. Gestionar con calidad es ser capaz de ofrecer un servicio o bien que satisfaga las necesidades y demandas de todo su grupo o colectivo, cumpliendo unos requisitos establecidos previamente sobre las características que han de tener los servicios prestad los. En sentido ultimo, la calidad de un servicio social pasa por el grado de satisfacción que genere y por el proceso de entrega (Ríos, 2008)

La calidad se convierte así, además de una exigencia, en un modo de gestión necesario y útil que va mucho más allá de certificar tal o cual actividad mediante una norma estandarizada. La calidad supone superar las inercias de la organización para entrar en una dinámica de mejora continua. Ahí las organizaciones sociales tienen un amplio campo de actuación que va a requerir sus mejores esfuerzos para responder a su propio compromiso de cambio y transformación social (Azúa, 2005).

El carácter desinteresado del voluntariado y el hecho de que a menudo muchas organizaciones de inspiración filantrópica se generan en base a distinciones que ponen el acento en el esfuerzo, el sacrificio que sus partícipes han llevado a cabo, ha impedido considerar la importancia que el tema de la calidad reviste. En muchas oportunidades, el impacto y efectividad de los programas sociales se basan en el poder del hacer que tienen los implementadores ( $\mathrm{Li}$ política de la organización, deben o se ven forzados en ciertas ocasiones a tomar decisiones de manera independiente, utilizar su propio criterio para generar respuestas pertinentes a determinados casos y en relación a los objetivos propuestos en las intervenciones. Así, los ejecutores de las políticas tienen una inmensa capacidad de discreción para cambiar la política o bien para ajustarse al diseño original de dichas políticas o seguir una agenda totalmente diferente. Intervenciones sociales diseñadas de esta manera, aparecen revestidas de opacidad, como un black box (Luhmann, 1998), dado el alto grado de discrecionalidad de los voluntarios ante las diferentes situaciones a las que se encuentran enfrentados. La discrecionalidad opera como factor reductor de las expectativas de ejecución de acciones y no por medio de mecanismos previstos por la propia estructura.

Estas características propias del voluntariado en organizaciones con un marcado cariz social y humanitario, no permite observarlo como una clásica membresía, tal como ha sido descrito en la teoría social de sistemas (Luhmann, 1998), ligado a la cadena decisional. Por ello que proponemos el concepto de cuasi-membresía para su descripción, en cuanto permite observar el carácter semiformal que lleva aparejado.

En cuanto estas organizaciones mantienen fuertes inconvenientes para reducir las expectativas del campo de actuación del voluntariado, la calidad de sus intervenciones sociales se encuentra restringida por las características de la interacción presencial entre los integrantes de la organización y las personas atendidas (Ríos, 2008:5) y, por lo tanto, no importa qué tan innovadora o participativa sea la propuesta de intervención o política social, gran parte de su éxito y calidad se encuentra constreñida por el trabajo realizado por la cuasi-membresía en su rol organizacional. Así, el principal escollo a superar para establecer criterios de calidad de las intervenciones sociales de este tipo, requiere que las organizaciones no solo consideren aquellos procesos de segundo orden, en el cual puedan observar, registrar y monitorear, sino también aquellos que se aplican en la interacción presencial con las personas atendidas por éstas. La calidad de los procesos desempeñados por la organización no es la misma que la que se debe verificar en la interacción (Ríos, 2008). Surge, así, un nuevo desafío para las organizaciones sociales: desarrollar estrategias de gestión de voluntariado.

En este sentido, sea cual sea el papel que desempeñe el voluntario en la organización, ésta debe prestar especial atención a este colectivo. La labor que realiza esta cuasi-membresía va más allá de la colaboración gratuita, por lo que es necesario dedicar tiempo y esfuerzo para garantizar que la relación entre la organización y el voluntariado se ajuste al requerimiento de las expectativas puestas en la estructura, velando por la coherencia con la consecución de la misión o sentido último de la entidad. La organización tiene así la responsabilidad de plantearse y gestionar el voluntariado de forma que se maximicen los preceptos organizativos de las personas voluntarias y de aquellos que reciben prestaciones de los procesos de intervención (Observatorio del Tercer Sector; 2009).

De igual forma, la gestión del voluntariado exige restringir el campo de actuación de sus expectativas, responsabilizándolos por su accionar, y utilizar los elementos motivacionales implicados en la caridad y la solidaridad, de manera que puedan reincorporarse en las intervenciones sociales desarrolladas. En este sentido, la responsabilidad del voluntario con su sociedad y el compromiso que busca adquirir para su cambio, debe coordinarse y alinearse con l compromiso de la organización. Esta debe contar con una orientación técnica desarrollada por su equipo profesional y los voluntarios deben incorporarse a esa orientación de manera que se limite su campo de actuación. La organización debe saber conjugar la libertad de actuación y de participación de los voluntarios y sus valores con el cumplimiento de sus objetivos, en el marco de su estrategia de intervención y su metodología de trabajo (Fundación Luis Vives, 2000:57)

Así, como señala Patti (2000) debido a que las organizaciones de servicios sociales dependen en gran medida del personal de primera línea, los gerentes de servicios sociales no solo deben desarrollar procesos en la agencia para incrementar el apoyo a objetivos comunes, sino que también deben articular los valores y objetivos de la organización que mspiran el compromiso moral de los voluntario que llevan a cabo los proceso de intervención de manera de alinear todos los elementos a la estrategia y objetivos institucionales. En ese sentido, deben considerarse estrategias de gestión de voluntariado como una manera de encuadrar a estos actores dentro de la misión y objetivos de la organización, as 
como también para poder capacitar y formar al voluntario de forma que éste ejecute adecuadamente y con calidad el servicio propuesto.

Organizaciones sociales chilenas y los desafíos pendientes para la gestión del voluntariado: hacia la definición de perfiles de voluntariado

Si bien los voluntarios no son algo nuevo dentro de la región, su perfil, sus motivaciones, el tipo de labor que desarrollan, su denominación, así como la percepción que de ellos tiene el resto de la sociedad, ha sufrido transformaciones con el correr de los tiempos. El voluntariado no siempre ha sido e mismo, sino que ha ido resignificando sus estructuras, objetivos, alcances, estrategias y visiones en la medida en que se han modificado los escenarios sociales donde ellas operan. Detrás de cada uno de los ciales donde e cambios hay una lectura de lo social que advierte al trabajo voluntario que "el país cambió" y que, por lo tanto, se requieren de nuevos mapas para navegar con éxito en lo social (Haz, 2007). Por esta razón aparece ahora el desafío imperante de que las organizaciones orientadas en la transformación social puedan contar con perfiles de voluntariado

Es por ello que una primera advertencia para el tratamiento del tema es comenzar reconociendo que cuando hablamos de "voluntariado" no nos estamo refiriendo a un conjunto social homogéneo, identificable y medible fácilmente sino, por el contrario a un conglomerado no fácilmente clasificable de situaciones en las que los seres humanos demuestran de disimiles maneras, su altruismo, su conciencia y de dismiles queras, su altruisno, su concienciay responsabilidad politica y social, sus pretensiones de ubicarse socialmente, o bien la única alternativ posible frente a situaciones como catástrofes o emergencias ambientales (Thompson \& Toro, 2000:1).

Frente a esta situación, muchos estudios afirman la imposibilidad de definir el voluntariado de un vez y para siempre, porque su característica fundamental es la de transformarse, adaptándose a las exigencias de una sociedad cada vez más compleja (Dávila, 2002: 4). De allí que no hay un "voluntariado" ideal con características comunes, sino diversas "situaciones" de voluntariado, cada una de ellas relacionada con el contexto en que se desenvuelve, los propósitos y enfoques de su acción (Thompson \& Toro, 2000: 1).

En este sentido, variados autores apuntan a la visibilización de la multiplicidad de voluntarios y profundizan indagando en las variables que generan estas diferencias, en cómo es que ellas se relacionan estableciendo nuevas distinciones, y en cómo es que ello influye en su gestión, tanto a nivel de organizaciones sociales como a nivel de políticas públicas (Reed \& Selbee, 2000; Vecina, 2001; Dávila, 2002. Dávila \& Chacón, 2004; Dávila \& Chacón, 2005; Esmond, 2004; Hustinx \& Lammertyn, 2004; Tarazona, 2004; Barreiro, 2009; Fouce, 2009; Brudney zona, 2004; Barre

En nuestro país, poco se ha hecho por identificar las dimensiones que se muestran útiles para diferenciar a ciertos grupos de voluntario. Si bien son variados los estudios que permiten caracterizarlos de forma perie intentan de forma empirica estudiar las relaciones entre distintas variables endógenas -sociodemográficas, motivacionales, etc.-, y su interrelación con diversas variables situacionales y contextuales (Rojas, 2004; Aravena, 2004; Sepúlveda \& Muñoz, 2009). A nivel nacional, podemos constatar que la mayor parte de los estudios parecen no considerar la diversidad y pluralidad a la que está sujeto el voluntariado, por lo que cuando describen a las muestras lo hacen de forma muy general y superficial. Un claro ejemplo es el estudio llevado a cabo por la División de Organizaciones Sociales del Gobierno de Chile con la colaboración de la Red "Voluntarios de Chile" el año 2009, realizado en el marco del Programa de Fomento al Voluntariado (DOS, 2009) Dicho estudio es un claro intento por recoger y dar cuenta de la pluralidad de voces tras el voluntariado, identificando diferencias asociadas a las variables motivación, características, valores y significados de la actividad voluntaria. Desde nuestra perspectiva, no obstante, no se logra caracterizar la complejidad y la diversidad en que las formas de voluntariado se expresan, concluyendo una vez más de forma generalizadora y volviendo a la idea de que el voluntariado es "uno". La diversidad del voluntariado se esconde así tras la generalización de los resultados.

Las consecuencias y repercusiones para las organizaciones sociales ante esta situación son variadas y evidentes. Primero, dado que no todos los voluntarios. Priener las das vo作 motivacionales, tampoco todas las organizaciones requieren del mismo perfil de voluntarios en su interior. Ante esta dificultad, ¿cómo reclutarlos de acuerdo al perfil requerido por la organización?

A diferencia de las organizaciones orientadas al lucro, que generalizan la atracción de la membresía mediante el requisito motivacional del dinero, identificando claramente el perfil y el conjunto de habilidades que este tiene o debiese tener, las orga- nizaciones sociales que trabajan con voluntarios desconocen los mecanismos motivacionales que logran desplegar interacciones vinculantes, dejando en un espacio de amplias expectativas la forma en que puede realizarse su reclutamiento. $\mathrm{Al}$ voluntariado no se le recluta solamente por su perfil profesional -en professonal -en la mayoria de losos, incluso, estos son aún estudiantes universitarios-, sino que en muchas ocasiones se apela a expectativas tan difusas como puede ser tan solo el interés por participar. Entonces, cabe plantear cuestionamientos como ¿a quiénes deben dirigirse las organizaciones?, tomando en consideración que no todos los voluntarios han de responder a los rec de la organización, y además, ¿cómo hacerlo? No contar con una adecuada identificación de perfiles de voluntarios, significa que las organizaciones presenten procesos de reclutamiento poco claros y procesos de selección inadecuados o poco efectivos. Una inadecuada actuación en la selección de candidatos puede repercutir de manera negativa en la organización, e implica una pérdida de los escasos recursos invertidos en procedimientos no efectivos, y, a su vez, un desvío de recursos que podrían ser destinados directamente a población que requiere una ayuda sincera y sentida. Tal como lo dice Dávila (2005:4), esto impide a las organizaciones rentabilizar las inversiones en recursos dirigidos a los mismos voluntarios y adoptar medidas para evitar los perjuicios que pueden sufrir tanto las organizaciones como los usuarios al recibir una prestación no profesionalizada.

En este escenario, un claro riesgo que podemos constatar es que el incremento sostenido de personas que quieren ser voluntarias no sea adecuadamente canalizado, lo que lleve apa ubiquen de forma apropiada en una organización ni en actividades concretas que les permitan satisfacer los requerimientos motivacionales que les llevaron a ser voluntarios. De esta forma, inician y terminan actividades voluntarias, con diversa variabilidad temporal en distintas organizaciones, sin conseguir acoplar temporalmente sus requerimientos motivacionales con las directrices organizacionales, hasta que finalmente renuncian, agobiadas por el sinsentido de dicha labor o rotan hacia otras organizaciones que puedan canalizar dichas expectativas una y otra vez. Todo esto lleva aparejada la decepción a nivel personal, la pérdida de recursos a nivel organizacional y la escasa atención profesional a una población altamente carenciada (Dávila, 2005:4)
En lo práctico, la falta de información y el vacío eórico existente respecto a la realidad de los voluntarios de nuestro país se traduce en problemas para la gestión del voluntariado en las organizaciones sociales, puesto que estas no tienen más remedio que apoyarse en continuos procesos de ensayo y error o abandonarse a la indefensión en la lucha contra las divesas difcultades que hallan en el recluta miento, selección, formación, supervisión y apoyo de los voluntarios, y a la escasa permanencia en las organizaciones de los mismos. Tal como indican Grossman \& Furano (2004), no importa qué tan bien intencionados sean los voluntarios, puesto que a menos que haya una infraestructura para apoyar dirigir sus esfuerzos, estos se volverán inadecuados o peor aún, se desencantarán del proceso y de su trabajo, retirándose, lo que podría dañar directamente a los destinatarios de los servicios ofrecidos. Ante este desafío imperante, es importante repensar las estrategias a través de las cuales se gestionan las organizaciones sociales.

No desconocemos el hecho de que puede haber ciertas organizaciones que prefieran no realizar priori el perfil del voluntario ni la definición explícita de sus tareas, pensando que el voluntario que acude a ella se adaptará inexorablemente al puesto escasamente definido. Pero en el fondo, desde nuestro punto de vista, bajo este planteamiento, existe una visión voluntarista del voluntariado; ellos pueden realizar cualquier cosa y con una única motivación Ayudar. Este sistema, que a corto plazo puede parecer factible, puede producir serios problemas especialmente a medio/largo plazo, al no realizar adecuadamente las tareas encomendadas producto de una sobrecarga de expectativas inadecuadamente tratadas. Por ello, para lograr una mayor efectividad es necesario especificar, tanto como sea posible, que perfil de voluntario se requiere para ejecutar las tareas que se les asignarán.

Desde nuestro punto de vista, el mensaje puede ser dicho de manera más fuerte, pero no más claro: solo en la medida en que las organizaciones sociales refuercen sus elementos de gestión a nivel personal de manera conjunta con su gestión a nivel organizativo y sectorial, serán capaces de afrontar los cambios del entorno y seguir contribuyendo a la mejora de la calidad de vida de las personas y comunidades que apoyan, y esto pasa, precisamente, por conta con perfiles de voluntariado y conocer, por lo tanto, quiénes son, así como también cuáles son sus intereses 


\section{Conclusiones}

Los sistemas sociales organizacionales identificados con el tercer sector juegan un rol preponderante en nuestra sociedad, destacado en la medida de que son capaces de instalar nuevos temas en la agend pública, proponiendo metodologías innovadoras para abordar problemas irresolutos y actuando como articuladores entre el Estado y la ciudadanía. De esta manera, muchas de estas organizaciones se han comprometido como socias estratégicas u operadoras de programas del sistema estatal, quedando en sus una gran parte de las políticas y programas sociales (Vaughan \& Arsneault, 2003)

Esta situación ha significado la validación y reconocimiento a nivel social de la labor de este tipo de sistemas organizacionales, aumentando también las exigencias de calidad y transparencia en las acciones que desarollon. En esta línea, siendo optimistas y en pleno desarrollo en nuestro país, tenemos que reconocer que dichas organizaciones han hecho avances en la profesionalización de sus actividades, en la planificación de sus tareas y estrategias, y en la diferenciación funcional de su estructura. En un contexto de transición hacia una sociedad cada vez más diferenciadu funcionalmente, se producen continuamente tensiones de integracion, tal como lo estamos observando con las demandas sentidas de los estudiantes universitarios por una mayor equidad y justicia social.

Es dentro de este contexto de mayor complejidad, que surge la necesidad por observar e intervenir los diseños con los cuales las organizaciones que utilizan voluntarios operan, tomando en cuenta la máxima responsabilidad que les cabe en su rol de implementadores y ejecutores cara a cara de las políticas o programas sociales. El éxito de una organización no lucrativa en el cumplimiento de sus fines y en su desarrollo como organización no reside tanto en factores económicos, metodológicos o tecnológicos -que también los incluye- sino en la importa sentido, parece relevante el debate sobre cuáles son as exigencias a las que se enfrentan las organizaciones sociales en términos de gestión de voluntariado para garantizar la calidad de sus servicios, así como también dar cuenta del rol que adquiere el Trabajo Social en esta tarea.

Si bien los desafíos son variados y múltiples, en esta ocasión hemos querido centrar el foco en la relevancia que cobra el contar con perfiles de voluntariado que nos permitan conocer a fondo quiénes son aquellos que componen este conglomerado, dando cuenta de sus motivaciones, intereses, significaciones, capacidades y necesidades.

Contar con este tipo de información resulta un factor clave para la formulación de estrategias de gestión de voluntariado al interior de organizaciones sociales. La relación entre organización y voluntariado es bidireccional, se trata de una relación de acoplamiento estructural que requiere de ambas partes. Por este motivo, la organización tiene la responsabilidad de plantearse y gestionar el voluntariado de forma que pueda asegurar su funcionamiento interno, sin perder calidad en su contraprestación social, en un doble propósito, por un lado de satisfacer las expectativas de las personas voluntarias de forma que puedan desarrollar su actividad y lograr sus objetivos y, por otro, de conseguir cumplir también los fines y la misión de la organización (Observatorio del Tercer Sector, 2009:22). Claramente, para que esto sea posible, es necesario conocer a cabalidad -o al menos con mayor profundidad que hasta ahora- a los voluntarios.

Siguiendo esta línea, el Trabajo Social interesado en estudiar organizaciones presenta altas posibilidades de aportar al tema de la gestión del voluntariado, en la medida en que sus intereses científicos y profesionales se dirijan a la observación y elaboración de propuestas de intervención que permitan identificar, gestionar y administrar las expectativas de los voluntarios, compatibilizándolas con las de la organización, alineando ello a la visión y misión de la misma, aportando a producir las transformaciones sociales a las cuales se encuentra abocado.

En este sentido, resulta primordial para un Trabajo Social interesado en distintos tipos de organizaciones, lograr un trabajo articulado, complementario y donde sea posible asegurar ciertos estándares de calidad o al menos disminuir los grados de discrecionalidad al intervenir en su realidad particular. Para lograr esto, se requiere de procesos de reclutamiento y selección adecuados y alineados con la estrategia organizacional, y para eso, contar con perfiles de voluntariado claros, resulta fundamental.

Finalmente, y en la medida en que las organizaciones sociales tengan acceso y generen conocimiento en relación a los voluntarios de manera conjunta con instancias gubernamentales u otro tipo de actores sociales interesados en la temática, será posible aportar a la gestión de calidad de las organizaciones sociales, aumentar su eficiencia, eficacia y fortalecer laporte del voluntariado a la sociedac, desarollando intervenciones sociales de calidad a quienes más lo requieren.

\section{Bibliografía}

ABOGAIR, X. (2006). Participación Ciudadana y Organizaciones No Gubernamentales (ONG). Serie En Foco $\mathrm{N}^{0}$ 86. Expansiva. Disponible en http:///www.expansipdf, el 15 agosto 2010

ARAVENA, M.S. (2004). Significados de la experiencia de voluntariado para las y los voluntarios de la Fundacion Gesta. Memoria pata psicólogo. Universidad de Chile. Santiago, Chile.

AZÚA, P. (2005). Retos y necesidades del tercer sector. Revista Española del Tercer Sector. Editada por Disponible en: http:/www fundacionluisives org rets/1/articulos/1740/index html el 22 de Junio 2011

ARREIRO, L. (2009). El voluntariado: entre la ciudadanía y la ideología. Rev. Katál. Florianópolis v. $12 \mathrm{~N}^{\circ} 2$ págs. 235-240. Jul./dic. 2009.

BRUDNEY, J. \& GRAZLEY, B. (2009). Moving Ahead or Falling Behind? Volunteer Promotion and Data Collection. Nonprofit Management \& Leadership, vol. 16, №.3, Spring.

CARITAS. (2011). Manual de formación de voluntariado. Disponible en la web: http://www.plataformavoluntariado.org/web/resources/index extraído el 24 de mayo.

COULSHED, V. (1998). La gestión del trabajo social, Editorial Paidos, Barcelona, España.

DÁVILA, Ma . C. (2002). La incidencia diferencial de los factores psicosociales en distintos tipos de voluntariado. Memoria presentada para optar al grado de Doctora en Psicología. Universidad Complutense de Madrid. Madrid, España.

DÁVILA, M.C., y CHACÓN, F. (2004). Factores psicosociales y tipo de voluntariado. Revista Psicothema, Vol. 16, No 4. Facultad de Psicología de la Universidad de
Oviedo. Asturias, España. Disponible en: http.//www. Oviedo. Asturias, España. Disponible en: http://www. psicothema.com/psicothema.asp?id=3044 el 22 Agos-
to 2011 .

DÁVILA, Ma. C. y CHACÓN, F. (2005) Variables socio demográficas y permanencia de diferentes tipos de voTo 89 . Con Rejo Genel de Colegios Ofiates de Psicologos. Madrid, España

DAVIS, K. (1999). El comportamiento humano en el trabajo. McGraw-Hill: México,

DIVISIÓN DE ORGANIZACIONES SOCIALES (DOS) (2009). La nueva fisonomía del voluntariado: actores, prácticas y desafíos. Resultados del primer catastro na. Ministerio Secretaría General de Gobierno. Santiago, Chile.

ESMOND, J. (2004). Developing the volunteer motivation inventory to assess the underlying motivational drives of volunteers in western Australia. Extraído el
22 agosto 2011: http://www.morevolunteers.com/resources/MotivationFinalReport.pdf

FLORES, R. (2003). Tercer sector, capital social y antropología cultural. En Revista Chilena de Antropología n¹7, Fac Cs.Soc. Universidad de Chile, págs, 33-46.

FOUCE, J. (2009). Voluntariado social en el siglo XXI ¿Movimiento social o instrumento neoliberal? En Intervencion Psicosocial: Revista sobre igualdad y calida de vida, Vol. 18, №. 2, págs. 177-190.

FUNDACIÓN LUIS VIVES. (2011). Propuestas de acción sobre el voluntariado y el marco financiero de Octubre, 2000. Extraído el 24 de mayo 2011: http:// www.fundacionluisvives.org/servicios/publicaciones/ detalle/2683.html.es

GROSSMAN, J B \& FURANO, K. (2004). Making the Most of Volunteers. Public/Private Ventures. En: Volunteer Management Practices and Retention of Volunteers The Urban Institute June.

HAGER, M. \& BRUDNEY, J. (2004). Volunteer Management: Practices and Retention of Volunteers. The

HAZ, A.M. (2011). Taller "El voluntariado y sus desafíos como intervención social". Proyectos Estudiantiles Nida Universitaria UC. Extraclo el 14 de Marzo. http.// mentos/apoyotallervoluntariado.pdf

HUSTINX, L., \& LAMMERTYN, F. (2004). The cultural bases of volunteering: Understanding and predicting attitudinal differences between Flemish Red Cross volunteers. Nonprofit and Voluntary Sector Quarterly, $\mathrm{N}$ 33, SAGE Journal Online. Extraído el 22 agosto 2011 http://nvs.sagepub.com/content/33/4/548

IBSEN, B. (1996). Changes in local voluntary asociatones in Denmark. Voluntas ingerizal Journal of University Press. Manchester, Inglaterra.

LIPSKY, M. (1980). Street-Level Bureaucracy: Dilemma of the Individual in Public Services. New York: Russell Sage Foundation.

LUHMANN, N. (1998). Sistemas sociales, lineamientos para una teoría general. Anthropos: Barcelona

OBSERVATORIO DEL TERCER SECTOR. (2009). Manual de Gestión del Voluntariado. Fundación La Caixa España.

OLATE, R. (2009). El Voluntariado Juvenil en América del Sur: un análisis de su orientación y formalización utilizando la teoria de los origenes sociales de la sociedad Universidad Católica de Chile. Santiago, Chile.

PATTI, R.J. (2006). The landscape of social welfare management. En: WUENSCHEL, Peter. The dimishing role of Social Work Administrators in Social Service 
Agencies: Issues for consideration. Administration in Social Work, Vol. 30, № 4. Pág. 12

PEROLD, H. y TAPIA, M.N. (editoras). Servicio Cívico y Voluntariado en Latinoamérica y el Caribe Solidario, Buenos Aires; Johannesburg Volunteer and Service Enquiry Southern Africa; St. Louis, Washington University, The Center for Social Development Washington DC, Innovations in Civic Participation. Pág. V-VI. Extraído el 14 de marzo de 2011: http:// www.service-enquiry.org.za

QUIJANO, S.; CORNEJO, J.M.; YEPES, M. \& FLORES, R. (2005). La calidad de los procesos y recursos humano (CPRH) como componente de la calidad del sistem humano de la organizacion. conceptualizacion y mediFacultad de Psicologí, Universidad de Bacelona 7 -

REDD, P. \& SELBEE, L. Distinguishing Characteristic of Active Volunteers in Canada. Nonprofit and Voluntary Sector Quarterly 2000; № 29. Extraído el 23 septiembre 2009: http://nvs.sagepub.com/cgi/conten abstract/29/4/571

RíOS, R. Capacidad organizacional y calidad de la Intervención Social. Perspectivas CEES-UC No 6. Documentos de trabajo. Centro de Estudios de Emprendimientos Solidarios, Pontificia Universidad Católica de Chile. Diciembre 2008, Santiago, Chile.

ROJAS, G. (2004). Fundación Gesta: Una aproximación Mad. No11 Septiembre 2004. Departamento de Antropología. Universidad de Chile. Extraido el 22 agosto 2011: www.revistamad.uchile.cl/11/paper07.pdf

SALAMON, L. et al. (2006). Estudio Comparativo del Sector Sin Fines de Lucro. Santiago, Chile. Abril.

SEPÚLVEDA, N \& MUÑOZ, A. Voluntariado y edades: Observaciones desde la juventud, la adultez y la vejez voluntaria de la ciudad de Santiago. Revista MAD, N 20, 2009. Universidad de Chile. Santiago, Chile.

TARAZONA, D. (2004). Acercamiento a la experiencia subjetiva del voluntariado social en organizaciones juveniles. Revista Flectrónica del Instituto Psicología Desarrollo, Página 2 de 12 Año I, Número 3, Diciembre. Disponible en: http://sicj.cepal.org/publicaciones/ ficha/:id=231 el 22 de Agosto 2011 .

THOMPSON, A \& TORO, L. (2000). "El voluntariado social en América Latina. Tendencias, influencias, espacios y lecciones aprendidas". Revista Sociedad Civil, Mexico. Documento incluido dentro de la Biblioteca Digital de la Iniciativa Interanticana de Capital soDesarrollo (BID).
VAUGHAN \& ARSNEAULT (2003). Nonprofit Federalism: Implications of the Partnership Between Governments and Not-for-Profit Organizations in the Association Annual Meeting. Chicago, Illinois.

VECINA, M.L. (2001). Factores psicosociales que influyen en la permanencia del voluntariado. Tesis doctoral no publicada, Universidad Complutense de Madrid.

VERNIS, A. Tensiones y retos en la gestión de las organizaciones no lucrativas. Revista Española del Tercer Sector. Editada por Fundación Luis Vives. N 1 1 Octubre - diciembre 2005. Extraido el 22 de junio 2011:

ZULUETA, S. (2003). La evolución del voluntariado en Chile entre los años 1990 y 2002. Tesis para optar al grado de Magíster en Sociología, Pontificia Universidad Catolica de Chile, Santiago, Chile.

\section{Las prácticas de participación política de dirigentes de Centros de Estudiantes de Trabajo Social en Universidades Privadas}

Practices of political participation within students representations organizations leaders'

Alejandra I. Santana L.

Directora de la Unidad de Mejoramiento de la Docencia, Universidad Autonoma de Chile. Trabajadora Social, Magister en Psicologia, Magister en Educación, Doctor @ en Ciencias de la Educación. Correos electrónicos: asantana@uc.cl / alejandra.santana@

Natalia Hernández M.

Academica del Departamento de Trabajo Social, Universidad Alberto Hurtado. Trabajadora Social, Magister en Trabajo Social, Estudiante de Doctorado en Trabajo Social. Correos electrónicos: nehernan@uc.cl / nhernand@uahurtado.d

\section{Resumen}

En un escenario sociocultural contemporáneo que ha transformado las diversas formas de participación del mundo juvenil, este articulo ofrece una aproximación teórica y empirica a las prácticas de participación politicas de dirigentes de Centros de Estudiantes de Trabajo Social en Universidades Privadas.

La investigación se orientó a conocer qué expresiones han ido conformando las prácticas políticas de estos jóvenes, indagando en: el significado de la imagen de dirigente, la participación y la práctica politica, las estrategias de participación, las apreciaciones referentes a los partidos politicos y la relación de los centros de estudiantes con sus respectivas federaciones en el contexto universitario. Para esto se trabajó mediante una metodologia cualitativa recogiendo los datos a través de focus group y analizándolos a través de matrices de contenidos temáticos.

Los principales hallazgos apuntan a reconocer que el rol de dirigente se relaciona con su identidad juvenil y por tanto con su habitus. Poseen una noción amplia de participación política relidadojua y pol ejercicio de la ciudadania la práctica política es diversa, buscando la participació en si misma Las estrategias usadas corresponden a acciones concretas y acotadas que buscaión participacín y representatividad de sus compañeros La relación con sus federaciones ouncan la paricipaín y repa

Palabras clave. Jóvenes, participación politica, universitarios.

Summary

In a socio cultural and political scenario that has changed the socio-political forms of participation of the youth world, this article offers a theoretical and empirical approximation to the practices of political participation of leaders of students' organizations in Private Universities in Chile.

The research focused on knowing what expressions have shaped the political practices of these young people, looking into. the meaning of the leader image, the participation and political practice, participation strategies, the significances concerning political parties and the relationship of students organizations with their students federations in the university context. For this purpose, we worked using a qualitative methodology, collecting data through focus groups and analyzing them using thematic content matrixes.

The main findings point to recognize that the role of leader is related to its youth identity and thus their habitus. They have a broad notion of participation policy, related to the exercise of citizenship. Political practice is diverse, and it's valued in itself. The strategies used correspond to concrete and delimited actions, which aim to promote participation and representation within other university students. Finally, the relationship with their federations or university support units can be stressful, evaluated by these leaders as mechanisms to control them.

Key words. Youth, political participation, university students. 